\title{
TISSUE-ENGINEERED BONE FOR TREATMENT OF COMBAT RELATED LIMB INJURIES
}

\author{
R.G. Vasyliev ${ }^{1,2}$, V.M. Oksymets ${ }^{2}$, A.E. Rodnichenko, , A.V. Zlatska ${ }^{1,2}$, O.S. Gubar ${ }^{2,3}$, \\ I.M. Gordiienko, ${ }^{2,}$, D.O. Zubov ${ }^{1,2, *}$ \\ ${ }^{1}$ State Institute of Genetic and Regenerative Medicine, National Academy of Medical Sciences of Ukraine, \\ Kyiv 04114, Ukraine \\ ${ }^{2}$ Biotechnology Laboratory ilaya.regeneration, Medical Company ilaya ${ }^{\circledR}$, Kyiv 03115, Ukraine \\ ${ }^{3}$ Institute of Molecular Biology and Genetics, NAS of Ukraine, Kyiv 03680, Ukraine \\ ${ }^{4}$ R. E. Kavetsky Institute of Experimental Pathology, Oncology and Radiobiology, NAS of Ukraine, \\ Kyiv 03022, Ukraine
}

\begin{abstract}
Aim: Based on our preliminary positive clinical results with use of cultured bone marrow-derived multipotent mesenchymal stem/stromal cells in traumatology, our aim was to develop living three-dimensional tissue-engineered bone equivalent transplantation technology for restoration of critical sized bone defects caused by combat related high energy trauma. Materials and Methods: To fabricate bone equivalent we used devitalized allogeneic bone scaffolds (blocks and chips) seeded with cultured autologous cells: bone marrow-derived multipotent mesenchymal stem/stromal cells in mix with periosteal progenitor cells and endothelial progenitor cells. Quality/identity of cell cultures was assured by donor and cell culture infection screening (immunofluorescence assay, polymerase chain reaction), flow cytometry (cell phenotype), karyotyping (GTG banding), functional assays (colony forming units analysis, multilineage differentiation assay). Bone defect treatment with bone equivalent application was fully completed in 39 combat-injured with $\mathbf{4 2}$ defects. New bone formation was assessed by the radiographic examination. Results: Casualties were included in a treatment program an average of 10.1 months after injury, provided the ineffectiveness of conventional surgery methods. All cell type cultures had a normal karyotype and appropriate phenotype, differentiation potential and functional properties, $\sim 30 \%$ colony forming units frequency and hadn't any signs of cell senescence. The fluorescein diacetate/propidium iodide combined staining and histology analysis of graft samples before transplantation showed their regular seeding with viable cells. Pathomorphological analysis of bone equivalent specimens 3-6 months post-op revealed the active remodeling processes and immature bone tissue formation. Bone defect restoration was observed 5-6 months post-op. Conclusion: The developed biotechnology of living three-dimensional tissue-engineered bone equivalent transplantation with overall effectiveness $90.4 \%$ allows restoring the bone integrity, forming new bone tissue in a site of bone defect, and significantly reducing the rehabilitation period of a patient.
\end{abstract}

Key Words: regenerative medicine, bone defects, tissue-engineered bone equivalent, human cell-based medicinal products, multipotent mesenchymal stromal/stem cells, endothelial progenitor cells.

The problems of high energy traumatic injuries of skeleton bones and the methods of their reconstruction in correlation with reparative osteogenesis are relevant for actual clinical and experimental traumatology and orthopedics. Temporary and economic costs for treating patients with alterations of reparative regeneration processes, especially in the high-energy mechanism of trauma, the complexity of their social adaptation, justify the need to search for innovative organ-saving technologies of regenerative medicine for bone integrity restoration. Particularly, it is actual the problem of treatment of combat related high energy bullet, shrapnel and blast injuries Ukrainian traumatologists faced with, seeing the military actions in eastern part of Ukraine.

Submitted: August 04, 2017.

*Correspondence: $\quad$ E-mail: zoubov77@yahoo.com Tel.: +380506970600

Abbreviations used: 3D TEBE - three-dimensional tissue-engineered bone equivalent; ADSCs - adipose-derived stem cells; ALP - alkaline phosphatase; BM-MSCs - bone marrow-derived multipotent mesenchymal stromal/stem cells; BMPs - bone morphogenetic proteins; CFU - colony forming units; EPCs - endothelial progenitor cells; FBS - fetal bovine serum; FDA - fluorescein diacetate; MSCs - multipotent mesenchymal stromal/stem cells; PBS - phosphate buffered saline; PI - propidium iodide; PPCs - periosteal progenitor cells.
The gold standard for extended bone defects' treatment is autologous osteoplastic surgery. Then there are methods of the defect filling with use of different osteoplastic materials, such as allogeneic and xenogeneic bone (e.g., demineralized bone matrix); synthetic and polymer materials; and using the method of compression-distraction osteosynthesis by llizarov. The choice of the method of plastic of the bone defect depends on its location and size.

The newest alternative to the conventional methods of bone plastic surgery is bone restoration methods based on the approaches of regenerative medicine. Currently, there are two approaches to bone regeneration. The first one is based on the delivery of high doses of bone morphogenetic and other factors to the defect zone, such as autologous platelet concentrate or recombinant bone morphogenetic proteins (BMPs). Marx et al. [1] first evaluated the use of platelet concentrate for bone healing in 1998, and his studies led to the development of an entire industry for its use in traumatology and orthopedics using platelet concentrator devices. BMPs were first proposed as bone morphogenes by Urist in 1965 [2]. In the late 1980s his team performed the first transplantation with their use [2, 3].

The second approach in bone regeneration is based on the delivery to the defect area of the critical mass 
of osteoprogenitor cells, which realize their trophic and integrative potencies in a wound for the formation of bone regenerate and new bone at final. For the first time Phemister in the 1940s used injections of bone marrow aspirate into the fracture non-unions [4]. In the 1960s Burwell proposed as an autograft of the iliac crest bone containing the bone marrow [5]. Subsequently, as a source of osteoprogenitor cells, a concentrated bone marrow-derived mononuclear fraction was used. Connolly first showed in 1998 that four-fold concentration of the mononuclear fraction of bone marrow significantly increases the degree of fusion of non-consolidated fractures in humans [6]. Muschler etal. in 2003 proposed the technique of saturation of allogeneic bone chips and crumbs with a bone marrow clot, both in animal models and in clinical practice of fracture consolidation [7, 8]. After preclinical studies of Muschler's technique, on a par with autograft application, the positive results of its effectiveness were confirmed by several completed multicenter clinical trials $[9,10]$. However, in some cases, neither the autograft nor Muschler's technique led to the formation of a dense bone in non-consolidated fractures revealed by theX-ray examination. Such dysfunction may be related to the individual osteogenic insufficiency of bone marrow cells, as well as to the compromised immune status of the patient. Thereby, the next step in bone regeneration was the use of immunomodulatory allogeneic cultured osteoprogenitor bone marrow-derived multipotent mesenchymal stromal/stem cells (BM-MSCs) [11-14]. Of note, the organ-saving bone regeneration technology Trinity Evolution $^{\text {TM }}$ (Orthofix, USA) based on Mushler's approach has been proposed to restore defects in casualties with combat related trauma obtained in Iraq and Afghanistan war conflicts. As an osteoinductive scaffold the authors from Walter Reed National Military Medical Center (Bethesda, USA) have used the allogeneic demineralized bone matrix chips pre-saturated with allogeneic cadaver mononuclear fraction of bone marrow containing uncultured and nonpurified MSCs [15]. Furthermore, in other studies for bone fracture consolidation, it was applied an alternative cell component - the adipose-derived MSCs, or adiposederived stem cells (ADSCs) [16, 17].

Considering the safety profile of cell therapies, it was explored a recent review analyzing 70 clinical studies worldwide with more than 1400 patients involved and treated with autologous or allogeneic adipose-derived MSCs. Thus, in case of allogeneic use it was shown the generation of specific antibodies towards donor cells in $19-34 \%$ of patients with not yet well known systemic impact. Concerning the oncological safety, only one case of breast cancer recurrence was noted out of 121 previous breast cancer patients within the 12-month followup periods. In other three trials examined with 32 previous prostate cancer patients involved, no cancer recurrences were found within 3 to 6 months follow-up period [18].

Our biotechnology approach to bone integrity restoration is based on the transplantation of a living threedimensional tissue-engineered bone equivalent (3DTEBE) developed by our R\&D group since 2004 in Ukraine [19, 20]. In its last modification the advanced 3D TEBE consists of decellularized allogeneic bone block of the required size and shape (for circular defects), or bone chips or crumbs (for tangential defects). These scaffolds are used as osteoinductive and osteoconductive carriers for cultured autologous osteoprogenitor BM-MSCs and periosteal progenitor cells (PPCs) intended for formation of the critical cell mass for new bone formation in situ, and endothelial progenitor cells (EPCs) from patient's own peripheral blood intended to enhance the vascularization and trophics of the manufactured 3D TEBE.

\section{MATERIALS AND METHODS}

Casualties. The forty seven casualties were included for a moment into the experimental treatment program assured by the Medical company ilaya ${ }^{\circledR}$ Bioethics Commission permission, patient's informed consent, local protocol for treatment approved by the Ministry of Health of Ukraine; 39 injured patients with 42 bone defects have been completely treated. Selected patients hadn't previous oncologic diseases reported and possessed circular and tangential critical sized defects (ones without spontaneous recovery throughout life, more than $3.0 \mathrm{~cm}$ ) of tubular bones and heel bone of various lengths and volume. The defects were allocated according to the Table 1. Assessment of the effectiveness of a treatment was performed by the X-ray examination over 3 and 6 months after grafting. In some cases, during the 3D TEBE adaptive resection surgery the extra fragments of the remodeling graft was taken, which was subsequently subjected to the pathomorphological analysis (e.g., 6 months after grafting, human cells). The sections were stained with hematoxylin \& eosin.

\section{Cell cultures}

Culture of the BM-MSCs. Heparinized bone marrow aspirate in a volume not more $5 \mathrm{ml}$ was obtained with an aspirating needle from the patient's iliac crest. Then, $1 \mathrm{ml}$ of aspirate was spread over culture flasks with an area of $175 \mathrm{~cm}^{2}$ (SPL, Korea). The primary culture was seeded into multi-flasks with an area of $875 \mathrm{~cm}^{2}$ (Corning, USA) for large scale cell expansion. The therapeutic dose of BMMSCs (50-300 $\cdot 10^{6}$ cells) was available over 30-40 days.

Culture of osteoprogenitor PPCs. A culture of PPCs was obtained from a minimal fragment of the fibula periosteum up to $1 \mathrm{~cm}^{3}$. The fragment was washed with antibiotic/antimycotic solution (BioWest, France) and poured into the $100 \mathrm{~mm}$ Petri dish (SPL, Korea). The primary culture was seeded into multi-flasks with an area of $875 \mathrm{~cm}^{2}$ (Corning, USA). The therapeutic dose of PPCs (20-60 $\cdot 10^{6}$ cells) was available over 30 days.

The growth medium for BM-MSCs and PPCs culturing consists of $\alpha-M E M, 10 \%$ fetal bovine serum (FBS), $1 \mathrm{ng} / \mathrm{ml}$ bFGF (Sigma-Aldrich, USA), $2 \mathrm{U} / \mathrm{ml}$ heparin sodium (Indar, Ukraine), $100 \mathrm{U} / \mathrm{ml}$ antibiotic-antimycotic solution (BioWest, France). Cell subculturing was carried out with a mixture of trypsin and EDTA solutions in a ratio of $0.05 \%: 0.02 \%$ in phosphate buffered saline (PBS) (Sigma-Aldrich, USA). The seeding density was $10^{3}$ cells per $1 \mathrm{~cm}^{2}$.

Culture of EPCs from the peripheral blood. EPCs were obtained from $20 \mathrm{ml}$ of heparinized venous blood of the 
patient. Each $5 \mathrm{ml}$ blood was spread per $75 \mathrm{~cm}^{2}$ culture flask (SPL, Korea) into the selective endothelium growth medium EGM-2 (Lonza, Italy). Single EPCs colonies from the primary culture were seeded in culture flasks with an area of $175 \mathrm{~cm}^{2}$ (SPL, Korea) to obtain a therapeutic dose. Cell subculturing was carried out with a mixture of trypsin and EDTA solutions in a ratio of $0.1 \%: 0.02 \%$ in PBS (Sigma-Aldrich, USA). The seeding density was $3 \cdot 10^{3}$ cells per $1 \mathrm{~cm}^{2}$. The therapeutic dose of EPCs (20-60 $\cdot 10^{6}$ cells) was available over 30 days.

All of the above cell types were grown in a multi-gas incubator (Binder CB 210, Germany) in an atmosphere of $5 \% \mathrm{CO}_{2}$ and $5 \% \mathrm{O}_{2}$ and a saturating humidity of $97 \%$.

All patients (peripheral blood - by immunofluorescence assay, polymerase chain reaction) and finalized cell cultures (by polymerase chain reaction) were screened for absence of HIV $1 / 2, \mathrm{HBV}, \mathrm{HCV}, \mathrm{HSV} 1 / 2$, CMV, EBV, Treponema pallidum and Mycoplasma ssp.

Functional assays for quality control. A set of quality control procedures were established to release personalized 3D TEBE as the human cell-based medicinal product. To determine the cell plating efficiency, colony forming units (CFU) analysis was performed according to the conventional protocol. For CFU staining, the cell colonies were fixed with cold ethanol and stained with azure-eosin by Romanowsky (Makrokhem, Ukraine) for 20 min [21]. Multilineage differentiation into the osteogenic and adipogenic directions was carried out according to the standard protocols [22]. Alkaline phosphatase (ALP) detection was carried out by the BCIP/NBT substrate assay (Sigma-Aldrich, USA). The normal karyotype of cultured cells was confirmed by GTG banding method [21].

\section{Directed multilineage differentiation assays}

Adipogenic differentiation was performed in the following medium: DMEM-HG ( $4.5 \mathrm{~g} / \mathrm{l})$ supplemented with $10 \%$ FBS, $1 \mu \mathrm{M}$ dexamethasone, $200 \mu \mathrm{M}$ indomethacine, $500 \mu \mathrm{M}$ isobutylmethylxanthine, $5 \mu \mathrm{g} / \mathrm{ml}$ insulin (Sigma-Aldrich, USA) and 5\% donor horse serum (BioWest, France). After 14 days the cells were fixed and stained. To detect the adipogenic differentiation, the cells were stained with $0.5 \%$ solution of Oil Red O dye for neutral intracellular lipids (Sigma-Aldrich, USA) [22].

Osteogenic differentiation was performed in the following medium: DMEM low glucose $(1.0 \mathrm{~g} / \mathrm{l})$ with $10 \%$ FBS, $100 \mathrm{nM}$ dexamethasone, $10 \mathrm{mM} \beta$-glycerophosphate and $50 \mu \mathrm{g} / \mathrm{ml}$ ascorbate-2-phosphate (Sigma-Aldrich, USA). After 21 days the cells were fixed and stained. To detect osteogenic differentiation, the cells were stained with $2 \%$ solution of Alizarin Red $\mathrm{S}$ dye for calcified extracellular matrix deposition (Sigma-Aldrich, USA) [22].

Endothelial cell tube formation assay. The assay measures the ability of endothelial cells, plated at subconfluent densities with the appropriate extracellular matrix support, to form capillary-like structures (tubes). The EPCs were added to the Matrige ${ }^{\mathrm{TM}}$ Matrix-coated (Corning, USA) well of 24-well plate (SPL, Korea) at the density $10^{3}$ cells per $200 \mu \mathrm{l}$, at a final medium volume of $200 \mu \mathrm{l}$ per $1 \mathrm{~cm}^{2}$ per well. Then they were incubated at $37^{\circ} \mathrm{C}, 5 \% \mathrm{CO}_{2}, 5 \% \mathrm{O}_{2}$ overnight in selective endothe- lium growth medium EGM-2 (Lonza, Italy). EPCs develop well-formed tube networks within 4-6 hours [23].

Flow cytometry. The number of positive and negative cells for corresponding markers (conventional, but not all-sufficient set) [14, 22] was measured for cultured BM-MSCs, PPCs and EPCs (at P2, $n=5$ per cell type) with use of flow cytometer BDFACSAria (BD Biosciences, USA). Staining with the monoclonal antibodies (PerCP-Cy5.5 mouse anti-human CD105, APC mouse anti-human CD73, FITC mouse anti-human CD90, PerCP-Cy5.5 mouse anti-human HLA-DR, PE-Cy-7 mouseanti-human CD31, PE mouse anti-human CD271, APC mouse anti-human CD34, FITC mouse antihuman CD45) was performed according to the manufacturer's instructions (BD Pharmingen, BD Horizon, USA).

3D TEBE manufacturing. As a scaffold for cells, a treated, cell-, DNA-free, allogeneic and non-immunogenic bone blocks of the required size and shape, bone chips and bone crust were used (A.A. Partners Ltd., Ukraine). In a first step the carrier was seeded directly with cells; in a second step the cell-seeded carrier was placed into the fibrin-derived hydrogel, also containing the cells. Cells were seeded over a carrier and into the fibrin hydrogel at a ratio of $2-5 \cdot 10^{6}$ cells per $1 \mathrm{~cm}^{3}$ scaffold. If the bone defect did not exceed $3 \mathrm{~cm}$, then the equivalent with only cultured BM-MSCs was used; if more than $5 \mathrm{~cm}-\mathrm{BM}-\mathrm{MSC}$ and PPCs were used at a ratio of $3: 1$; if more than $7 \mathrm{~cm}-$ BM-MSCs, PPCs and EPCs were applied in a ratio of 3:1:1. The appropriately seeded 3D TEBE before the transplantation was incubated for 5-14 days according to the size and volume in the BM-MSCs growth medium in a multi-gas incubator (Binder CB 210, Germany) in an atmosphere of $5 \% \mathrm{CO}_{2}$ and $5 \% \mathrm{O}_{2}$ and a saturating humidity of $97 \%$. The quality and regularity of seeding of the equivalent fragment with cells were confirmed by the combined staining with fluorescein diacetate/propidium iodide (FDA/PI) fluorescent dyes (Sigma-Aldrich, USA).

Microscopy. Inverted fluorescent microscope AxioObserver A1 equipped with digital camera AxioCamERc 5s and ZEN 2012 software (Carl Zeiss, Germany) were applied.

Statistics. The data are presented as mean and SEM $(M \pm m)$. Statistical significance was estimated using the Student's $t$-criterion.

\section{RESULTS AND DISCUSSION}

The gold standard for critical sized bone defect restoration is autologous bone graft transplantation. Although this surgery possesses its drawbacks, such as morbidity, risk of donor wound contamination and non-healing, risk of lysis of transplanted bone autograft. As a substantial alternative for autologous bone grafting we developed the regenerative medicine organ-saving biotechnology of the 3D TEBE transplantation.

The total number of treated casualties using tissue engineering approach was 47 (male -46 , female 1). The treatment was completed in 39 patients. Forty seven wounded persons had critical sized bone defects of different location (Table 1). Eight patients are 
in the treatment course. The age of the patients was $21-48$ years (the average age is 33.9 years). Before entering the clinic course, the wounded persons were subjected to 2-27 surgeries (an average of 5.7 surgeries). The patients came in treatment 2-22 months (an average of 10.1 months) after the combat related injury and unsuccessful surgeries in other foreign and Ukrainian hospitals. Most casualties had received a prescription to the limb amputation.

Table 1. Total number of patients and allocation of critical sized bone defects in casualties subjected to 3D TEBE transplantation

\begin{tabular}{lccccccc}
\hline $\begin{array}{c}\text { Bone defect } \\
\text { allocation }\end{array}$ & \multirow{2}{*}{ Arm } & \multirow{2}{*}{ Forearm } & Femur & Shin & Heel & \multicolumn{3}{c}{ Total } \\
\cline { 5 - 8 } Proximal third & 4 & - & 2 & 6 & - & 12 & 25.5 \\
Diaphysis & 5 & 5 & 8 & 10 & - & 28 & 59.6 \\
Distal third & 3 & - & - & 1 & - & 4 & 8.5 \\
Calcaneus & - & - & - & - & 3 & 3 & 6.4 \\
Total & 12 & 5 & 10 & 17 & 3 & 47 & 100.0 \\
\hline
\end{tabular}

Transplantation of 3D TEBE in the form of bone blocks was performed in 10 wounded, in the form of chips - in 22 wounded, and in the form of bone blocks and chips -13 casualties. The volume of the transplant ranged from 10 to $180 \mathrm{~cm}^{3}$ (on the average $40.4 \mathrm{~cm}^{3}$ ). The average density of graft seeding was 4.7 million cells per $1 \mathrm{~cm}^{3}$. The manufactured bone equivalent was homogenously seeded with cells after incubation on a scaffold as proved by FDA/PI combined staining procedure. The cell cultures were appropriately characterized for their identity, purity, potency, viability and suitability for the intended use [14, 22, 23]. All cell cultures had a normal karyotype and phenotype, differentiation potential and functional properties, 30\% CFU frequency and hadn't any signs of cell senescence.

Cultured BM-MSCs and PPCs were positive for conventional (but not unique) stromal markers CD105, CD90, CD73, negative (<2\%) by hematopoietic markers CD34, CD45, and negative for MHC class II molecules HLA-DR (Table 2). Cultured PPCs were also positive for CD271 (a versatile pre-culture marker, reported to be expressed only on isolated, but not cultured adult MSCs to selectively isolate and expand the adult stromal cells with both immunosuppressive and lympho-hematopoietic engraftment-promoting properties) [24]. Cultured EPCs were highly positive for CD31 (key endothelial marker), CD105 and CD73, positive for CD34 ( 35\%), and negative for CD45 and HLA-DR. It is interesting to observe the expression of the CD90 stromal marker on in vitro expanded EPCs being low as 3.4\% (see Table 2).

Table 2. Positive and negative cell surface markers of all cultured cell types involved in 3D TEBE manufacturing (determined by flow cytometry). Data presented as a percentage of parent population, \%

\begin{tabular}{ccccccccc}
$\begin{array}{c}\text { Cell cul- } \\
\text { ture }\end{array}$ & CD105 & CD90 & CD73 & CD271 & CD31 & CD34 & CD45 & $\begin{array}{c}\text { HLA- } \\
\text { DR }\end{array}$ \\
\hline BM-MSC & $99.7 \pm$ & $95.6 \pm$ & $99.8 \pm$ & ND & ND & $0.4 \pm$ & $0.4 \pm$ & $0.8 \pm$ \\
& 0.1 & 1.2 & 0.0 & & & 0.1 & 0.1 & 0.3 \\
PPCs & $97.5 \pm$ & $97.7 \pm$ & $99.4 \pm$ & $29.7 \pm$ & ND & $1.1 \pm$ & $0.3 \pm$ & $1.6 \pm$ \\
& 1.7 & 1.4 & 0.4 & 3.7 & & 0.6 & 0.1 & 0.6 \\
EPCs & $99.2 \pm$ & $3.4 \pm$ & $99.6 \pm$ & ND & $99.2 \pm$ & $35.0 \pm$ & $0.7 \pm$ & $2.7 \pm$ \\
& 0.3 & 1.4 & 0.2 & & 0.1 & 11.7 & 0.2 & 1.2 \\
\hline
\end{tabular}

Note: at P2, $\mathrm{n}=5$; ND - not determined.

Of note, only PPCs, but not BM-MSCs cultures at their basal culture level were ALP positive. Moreover, the PPCs cultures failed their adipogenic differen- tiation assay after 14 days of induction. It means the strong osteogenic commitment of this cell type without directed osteogenic induction in vitro (Fig. 1).

Histological analysis of 3D TEBE biopsies taken during graft adaptation resection surgery 3-6 months after transplantation showed graft intensive remodeling and immature bone tissue formation (Fig. 2).

Restoration of the bone defects was observed after 5-6 months post-op and evaluated by radiographic examination in 39 patients (42 critical sized bone defects). The results of the treatment are done in the Table 3 and considered as the following: Good - the formation
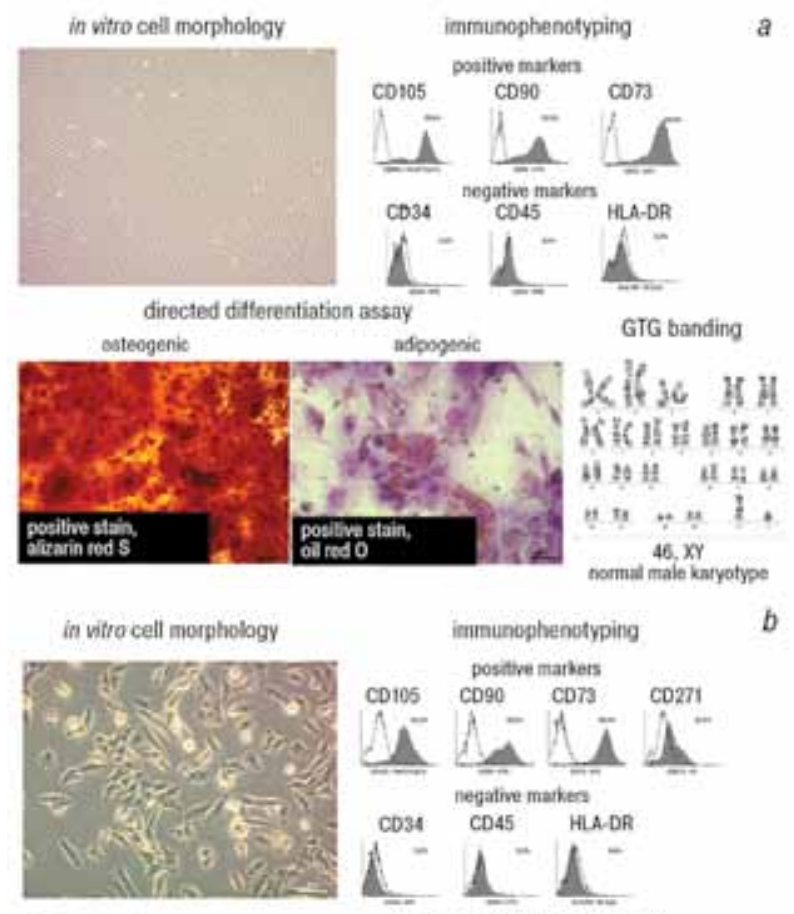

b
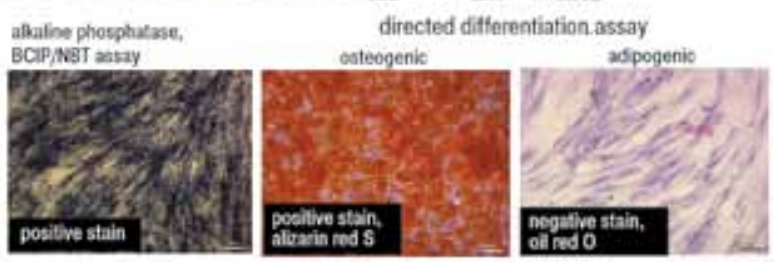

in vifro cell marphology

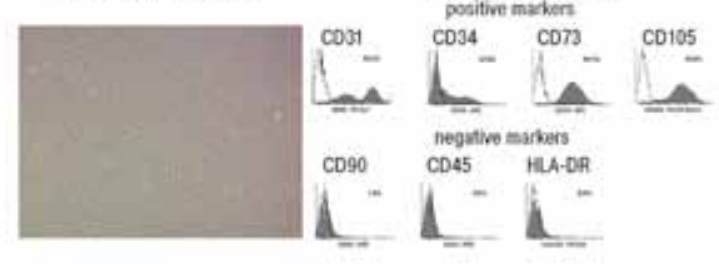

capillary-ike structures (tubes) formation in 3D matrix
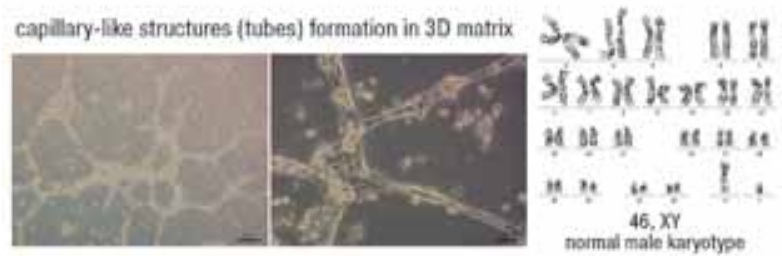

Fig. 1. Cell cultures' functional assays for QC: $a-B M-M S C s$ (morphology, immunophenotype, karyotype, directed differentiation assay); $b-$ PPCs (morphology, immunophenotype, directed differentiation assay, BCIP/NBT for ALP detection); $c-$ EPCs (morphology, immunophenotype, karyotype, endothelial cell tube formation assay in Matrige ${ }^{\mathrm{TM}}$ Matrix) 


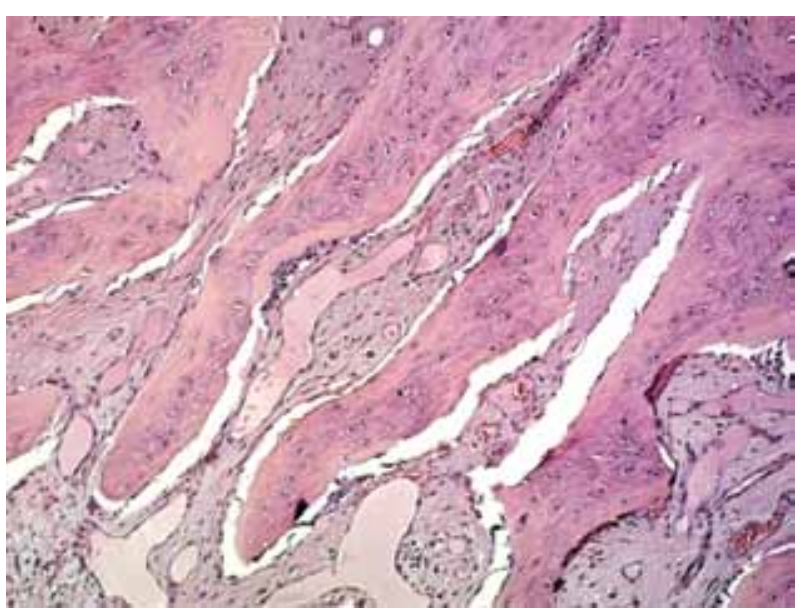

Fig. 2. Histological analysis of grafted $3 D$ TEBE. Patient $\boldsymbol{M}$., male, 25; bullet wound, right clavicle subtotal defect $(5.0 \mathrm{~cm})$. Remodeling and immature bone (osteoid) formation 6 months post-transplantation; biopsy taken during graft adaptation resection surgery; H\&E stain, $100 \times$ magnification

of bone tissue with the restoration of the integrity of bone segments of a limb within 4-6 months post-op (Fig. 3); Satisfactory - patients who had partial graft lysis in the transplantation area, but bone was formed provided the functional capacity of injured limb; patients who experienced a delay (more than 6 months) for bone tissue formation post-op; Unsatisfactory - patients who had a complete lysis of the transplanted bone equivalent. Thereof, the overall effectiveness of treatment of critical sized bone defects with 3D TEBE was $90.4 \%$.

Table 3. The results of completed critical sized bone defects' treatment with use of 3D TEBE

\begin{tabular}{lcc}
\hline \multicolumn{1}{c}{ Results } & $\mathrm{n}$ & $\%$ \\
\hline Good & 30 & 71.4 \\
Satisfactory & 8 & 19.0 \\
Unsatisfactory & 4 & 9.6 \\
Total & 42 & 100 \\
\hline
\end{tabular}

Note: 39 patients with 42 critical sized bone defects.

The biomedicinal product based on the human cells developed by our group can be attributed, depending on the main regulatory systems (European Medicines Agency - Committee for Advanced Therapies, EU vs Food and Drug Administration, USA) either to the (1) "Human Cell-Based Medicinal Products" - in terms of the European Medicines Agency [25], or to the (2) "Human cells, tissues, and cellular and tissue-based products", those in terms of the Food and Drug Administration [26]. Seeing the signing of the Association agreement between Ukraine and the European Union in 2014, our legislation and regulatory on the transition of biomedicinal product based on human cells into the clinical practice should be gradually harmonized with the EU legislation.

Based on our developed method for treatment of critical sized bone defects with the use of regenerative medicine approaches, we have planned and registered the clinical study at the International Registry ClinicalTrials.gov entitled "3D Tissue Engineered Bone Equivalent for Treatment of Traumatic Bone Defects (3D TEBE)"; ClinicalTrials. gov Identifier: NCT03103295. ClinicalTrials. gov is a registry and results information database of clinical research studies
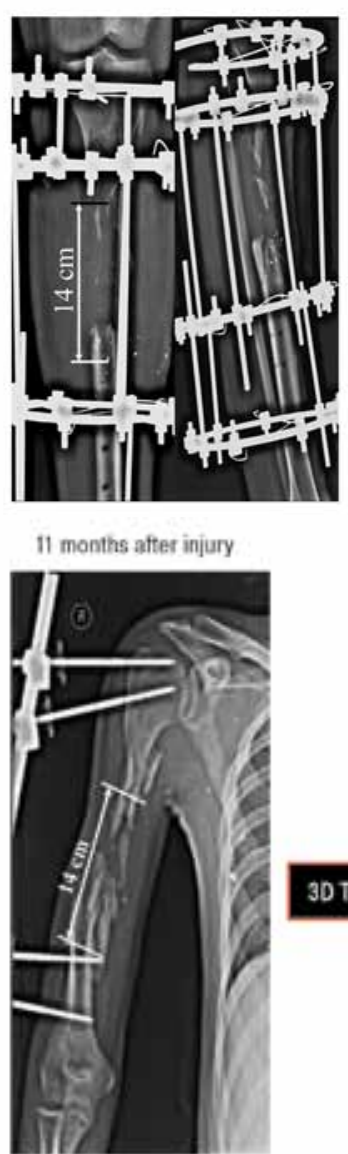

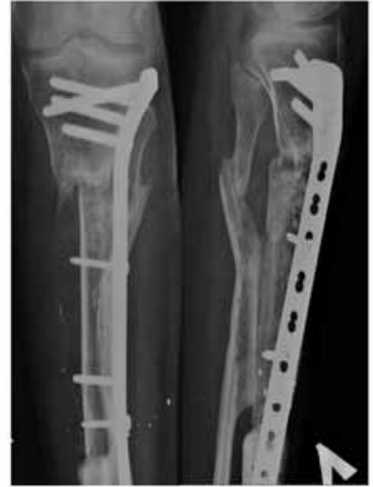

6 months after transplantation $b$
Fig. 3. Clinical cases of 3D TEBE application in casualties; $14 \mathrm{~cm}$ critical sized circular defects of diaphysis; semi-annual results after transplantation (X-ray examination): $a-$ patient $\boldsymbol{A}$. male, 27 , bullet wound, left tibia; $b$ - patient $\boldsymbol{B}$., male, 37 , bullet wound, right arm

sponsored or funded by a broad range of public and private organizations around the world [27].

Thereof, the developed regenerative medicine approach to organ-saving transplantation of the living 3D TEBE, with the overall effectiveness of $90.4 \%$, allows restoring the bone integrity, forming new bone tissue in a site of bone defect of critical size, and significantly reducing the rehabilitation period of a patient.

\section{REFERENCES}

1. Marx RE, Carlson ER, Eichstaedt RM, et al. Plateletrich plasma: growth factor enhancement for bone grafts. Oral Surg Oral Med Oral Pathol Oral Radiol Endod 1998; 85: 638-46.

2. Urist MR. Bone. Formation by autoinduction. Science 1965; 150: 893-9.

3. Johnson EE, Urist MR, Finerman GA. Bone morphogenetic protein augmentation grafting of resistant femoral nonunions. A preliminary report. Clin Orthop 1988; 230: 257-65.

4. Phemister D. Treatment of ununited fractures by onlay bone grafts without screw or tie fixation and without breaking of the fibrous union. J Bone Joint Surg 1947; 29: 946-60.

5. Burwell RG. Studies in the transplantation of bone VII. The fresh composite homograft-autograft of cancellous bone: an analysis of factors leading to osteogenesis in marrow transplants and in marrow-containing bone grafts. J Bone Joint Surg Br 1964; 46: 110-40. 
6. Connolly JF. Clinical use of marrow osteoprogenitor cells to stimulate osteogenesis. Clin Orthop Rel Res 1998; 355: S257-66.

7. Muschler GF, Nitto H, Matsukura Y, et al. Spine fusion using cell matrix composites enriched in bone marrow-derived cells. Clin Orthop Rel Res 2003; 407: 102-18.

8. Muschler GF, Nitto H, Matsukura Y, et al. Selective retention of bone marrow-derived cells to enhance spinal fusion. Clin Orthop Rel Res 2005; 432: 242-51.

9. Lieberman I. Local cell delivery strategies. Bone Summit, Cleveland: OH, 2004.

10. Wang JC, Youssef JA, Lieberman IH, et al. A prospective, multicenter study of selective osteoprogenitor cell retention for enhancement of lumbar spinal fusion. IMAST, Banff, Canada, 2005.

11. Jaiswal N, Haynesworth SE, Caplan AI, et al. Osteogenic differentiation of purified, culture-expanded human mesenchymal stem cells in vitro. J Cell Biochem 1997; 64: $295-312$.

12. Muschler GF, Boehm C, Easley K. Aspiration to obtain osteoblast progenitor cells from human bone marrow: the influence of aspiration volume. J Bone Joint Surg Am 1997; 79: $1699-709$.

13. Chatterjea A, Meijer G, van Blitterswijk C, de Boer J. Clinical application of human mesenchymal stromal cells for bone tissue engineering. Stem Cells Int 2010; 11: 1-12.

14. Dominici M, Le Blanc K, Mueller I, et al. Minimal criteria for defining multipotent mesenchymal stromal cells. The International Society for Cellular Therapy position statement. Cytotherapy 2006; 8: 315-7.

15. Fleming ME, Bharmal H, Valerio I. Regenerative medicine applications in combat casualty care. Regen Med 2014; 9: 179-90.

16. Cowan CM, Shi YY, Aalami OO, et al. Adipose-derived adult stromal cells heal critical-size mouse calvarial defects. Nat Biotechnol 2004; 22: 560-7.

17. Betz O, Vrahas M, Baltzer A, et al. Gene transfer approaches to enhancing bone healing. In: Bone Regeneration and Repair. JR Lieberman, GE Friedlaender, eds. Totowa, NJ: Humana Press, 2005: 158-62.

18. Toyserkani NM, Jørgensen MG, Tabatabaeifar S, et al. Concise review: a safety assessment of adipose-derived cell therapy in clinical trials: a systematic review of reported adverse events. Stem Cells Transl Med 2017; 6: 1786-94.

19. Oksymets V, Zubov D, Vasyliev R, et al. Use of cultured autologous BM-MSCs for altered post-traumatic reparative osteogenesis treatment: nonrandomized clinical trial. World Conf on Regenerative Medicine, Leipzig, 21-23 October 2013: Abstr. Regenerative Med 2013; Suppl: 47-8.

20. Oksymets V, Zubov D, Vasyliev R, et al. Clinical use of human-cultured, autologous, bone marrow-derived MSCs for treatment of the femoral head avascular necrosis. World Conference on Regenerative Medicine, Leipzig, 21-23 October 2013: abstracts. Regenerative Med 2013; Suppl: 226-7.

21. Freshney RI. Culture of animal cells: a manual of basic technique. NY: A.R. Liss, 1983. 295 p.

22. Prockop DJ, Phinney DG, Bunnell BA. Mesenchymal stem cells: methods and protocols. Totowa, NJ: Humana Press, 2008. 192 p.

23. Davis G, Senger D. Endothelial extracellular matrix: biosynthesis, remodeling, and functions during vascular morphogenesis and neovessel stabilization. Circ Res 2005; 97: 1093-107.

24. Alvarez-Viejo M, Menendez-Menendez Y, OteroHernandez J. CD271 as a marker to identify mesenchymal stem cells from diverse sources before culture. World J Stem Cells 2015; 7: 470-6.

25. EMEA/CHMP/410869/2006. EMEA/CHMP Guideline on Human Cell-Based Medicinal Products.

26. 21CFR1271. Code of Federal Regulations. Title 21 Food and drugs. Chapt. I - Food and Drug Administration: Department of health and human services. Subchapt. L - Regulations under certain other acts administered by the Food and Drug Administration. Part 1271 - Human cells, tissues, and cellular and tissue-based products: Subpart D - 